\title{
The Effective Pore Diameter of a Three-Dimensional Numerical Model for Estimating Heat and Fluid Flow Characteristics in Metal Foams
}

\author{
Fujio Kuwahara* and Yutaro Fumoto
}

Department of Mechanical Engineering, Shizuoka University, Hamamatsu, Japan

\begin{abstract}
A three-dimensional numerical model is proposed to determine heat and fluid flow characteristics in metal foams. A series of full three-dimensional numerical calculations was performed to reveal complex three-dimensional velocity, pressure and temperature fields within three-dimensional porous structures of the metal foams. These numerical results are processed to obtain the macroscopic characteristics such as the permeability, inertia, dispersion and interstitial heat transfer coefficients. An effective pore diameter concept has been proposed to correlate the resulting heat and fluid flow characteristics with available empirical correlations.
\end{abstract}

Keywords: Porous media, Numerical simulation, Metal foam, Pressure drop, Permeability, Convection, Periodic boundary condition.

\section{INTRODUCTION}

Metal foam materials have complex and irregular structure, and there are many pores with different sizes (see Fig. 1). They have meandering flow paths and large surface area as well as low density. And they have high thermal conductivity. Because of these characteristics, high performance of heat transfer between the fluid and solid phases can be achieved using such foams with high permeability. Therefore, metal foam materials can be used for high performance heat exchangers. Heat and fluid flow characteristics must be explored to optimize these equipments. Recently, considerable attention has been directed to numerical investigations [1-4]. However, heat transfer characteristics in metal foams have not been fully investigated numerically. Moreover, there is no general method to determine the characteristic length of three-dimensional numerical models yet.

In this study, we shall conduct a numerical study on heat and fluid flow in metal foam using a three-dimensional numerical model of periodical structure. In order to capture irregularities in real foams, quantities calculated at specific flow angles are ensemble-averaged over the flow angle. A rational way to evaluate the effective pore diameter, which is used as the characteristic length of present three-dimensional numerical model, is proposed. Permeability, Forchheimer coefficient, thermal dispersion and volumetric heat transfer coefficient are determined by spatially averaging microscopic numerical results. The validity of the present numerical model and the effective pore diameter are examined by comparing the numerical results with available empirical correlations. Furthermore, an interesting relationship between the thermal dispersion conductivity and the volumetric heat transfer coefficient is elucidated.

*Address correspondence to this author at the Department of Mechanical Engineering, Shizuoka University, Hamamatsu, Japan;

Tel: 81-53-478-1046; E-mail: fsk-dara@krf.biglobe.ne.jp

\section{NUMERICAL MODEL AND METHOD}

We shall consider heat and fluid flow through a foam heated at constant temperature $T_{s}$ as shown in Fig. (2a). Governing equations for the fluid phase are given as follows.

$\nabla \cdot \vec{u}=0$

$\rho_{f}(\vec{u} \cdot \nabla) \vec{u}=-\nabla p+\mu \nabla^{2} \vec{u}$

$\rho_{f} c_{p f} \vec{u} \cdot \nabla T=k_{f} \nabla^{2} T$

where the subscripts $f$ denotes the fluid phase. $\rho, c_{p}, k$ and $\mu$ are density, specific heat, thermal conductivity and viscosity, respectively. Since the structure is periodic, numerical domain can be limited to a structural unit (see Fig. (2b)) exploiting periodic boundary conditions. The macroscopic flow direction is set in terms of $\alpha$ and $\beta$ as shown in Fig. (2c). Calculations are performed for various sets of $\alpha$ and $\beta$, and these results are ensemble-averaged to determine the representative characteristics. The boundary and compatibility conditions are set as follows [5].

Solid walls:

$\vec{u}=\overrightarrow{0}, T=T_{s}$

Boundaries:

$\left.\vec{u}\right|_{x=0}=\left.\vec{u}\right|_{x=H},\left.\quad \vec{u}\right|_{y=0}=\left.\vec{u}\right|_{y=H},\left.\quad \vec{u}\right|_{z=0}=\left.\vec{u}\right|_{z=H}$

$\left.\int_{0}^{H} \int_{0}^{H} u\right|_{x=0} d y d z=\left.\int_{0}^{H} \int_{0}^{H} u\right|_{x=H} d y d z=H^{2}|\langle\vec{u}\rangle| \cos \alpha \cos \beta(6 \mathrm{a})$

$\left.\int_{0}^{H} \int_{0}^{H} v\right|_{y=0} d x d z=\left.\int_{0}^{H} \int_{0}^{H} v\right|_{y=H} d x d z=H^{2}|\langle\vec{u}\rangle| \sin \alpha$

$\left.\int_{0}^{H} \int_{0}^{H} w\right|_{z=0} d x d y=\left.\int_{0}^{H} \int_{0}^{H} w\right|_{z=H} d x d y=H^{2}|\langle\vec{u}\rangle| \cos \alpha \sin \beta(6 \mathrm{c})$ 


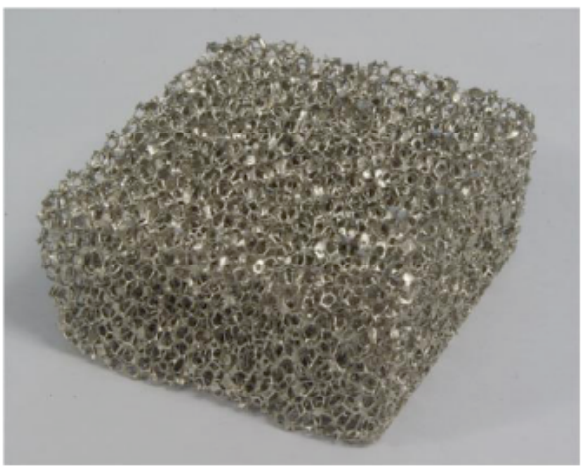

(a) Metal foam material

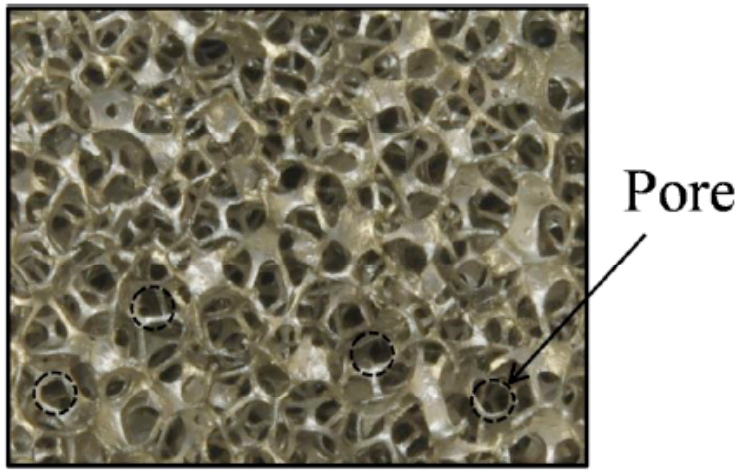

(b) Microscopic image of metal foam

Fig. (1). Foam material.

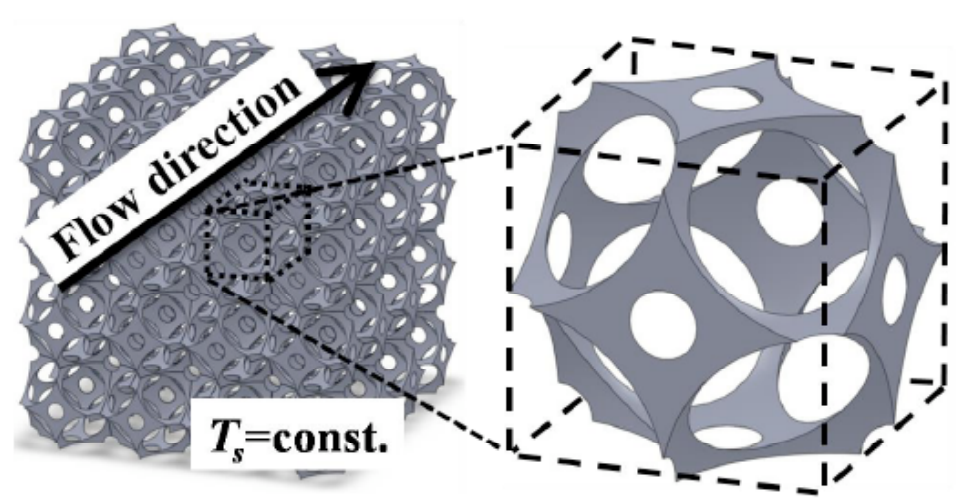

(a) Three dimensional numerical model (b) A structural unit

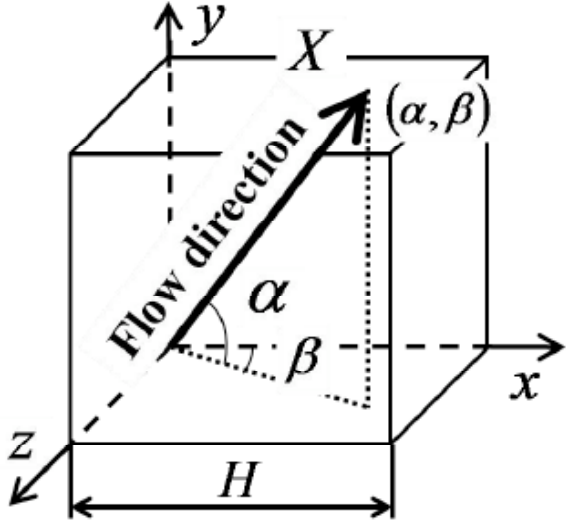

(c) Macroscopic flow direction

Fig. (2). Numerical model.
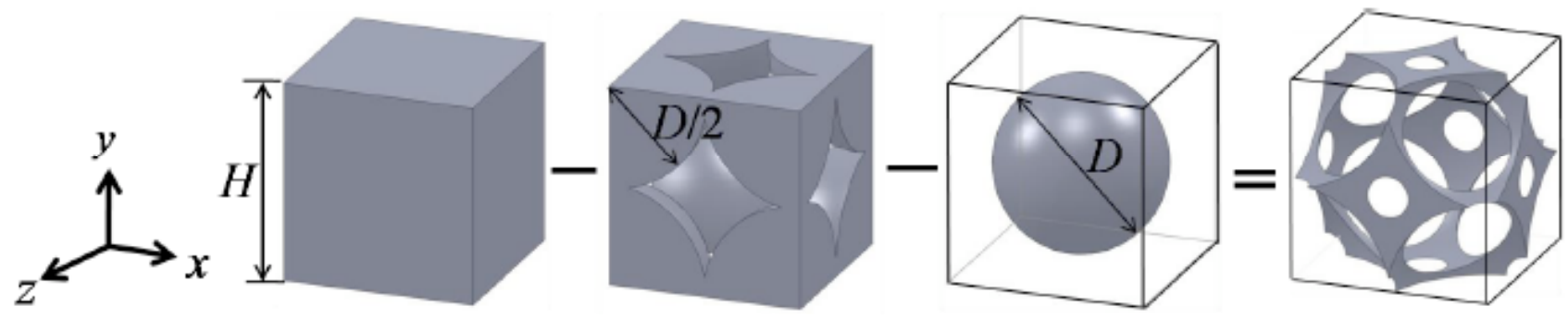

Fig. (3). Generation of a structural unit.

$$
\begin{aligned}
& \left.\left(T-T_{s}\right)\right|_{x=0}=\left.\tau_{x}\left(T-T_{s}\right)\right|_{x=H} \\
& \left.\left(T-T_{s}\right)\right|_{y=0}=\left.\tau_{y}\left(T-T_{s}\right)\right|_{y=H} \\
& \left.\left(T-T_{s}\right)\right|_{z=0}=\left.\tau_{z}\left(T-T_{s}\right)\right|_{z=H} \\
& \tau_{x}=\tau^{\cos \alpha \cos \beta /(\cos \alpha \cos \beta+\sin \alpha+\cos \alpha \sin \beta)} \\
& \tau_{y}=\tau^{\sin \alpha /(\cos \alpha \cos \beta+\sin \alpha+\cos \alpha \sin \beta)} \\
& \tau_{z}=\tau^{\cos \alpha \sin \beta /(\cos \alpha \cos \beta+\sin \alpha+\cos \alpha \sin \beta)} \\
& \tau=\frac{\left.\left(T-T_{s}\right)\right|_{x=0, y=0, z=0}}{\left.\left(T-T_{s}\right)\right|_{x=H, y=H, z=H}}
\end{aligned}
$$

All calculations are conducted for a structural unit using grid nodes $151 \times 151 \times 151$, to ensure grid independency. Fig. (3) shows a procedure to generate a structural unit. A cubic space $(H \times H \times H)$ is subtracted by spheres $(D)$ placed at vertices and the center of the cube. All structural units used in calculations are shown in Fig. (4). Present threedimensional numerical model has the same structure with Kelvin model [6]. There are two kinds of pores A and B with different sizes. But only pore A is in low porosity. The porosity is set from $\varepsilon=0.839$ to $\varepsilon=0.964$ by changing $D$.

In general way, a diameter of pore is determined by image analysis [7] or rational method [8]. From these methods, a pore diameter is not a pure diameter but an effective diameter. Thus, the effective pore diameter to be used for a characteristic length may be evaluated from

$d_{m}=\sqrt{\frac{4 \varepsilon}{n \pi}}$ 


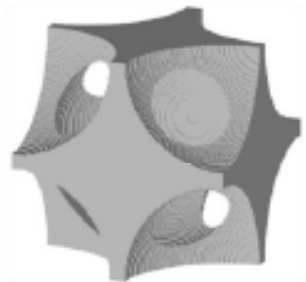

(a) $\varepsilon=0.839$

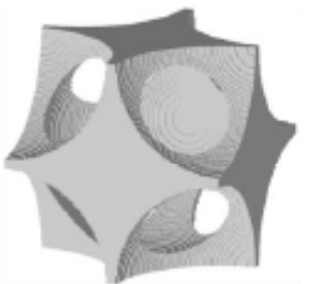

(b) $\varepsilon=0.875$

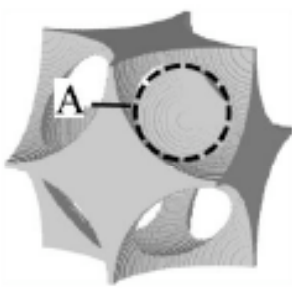

(c) $\varepsilon=0.909$

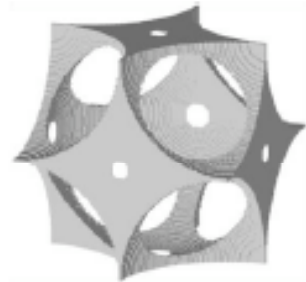

(d) $\varepsilon=0.939$

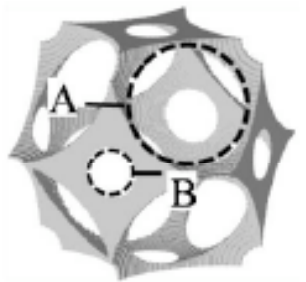

(e) $\varepsilon=0.964$

Fig. (4). Bird's view of structural units.

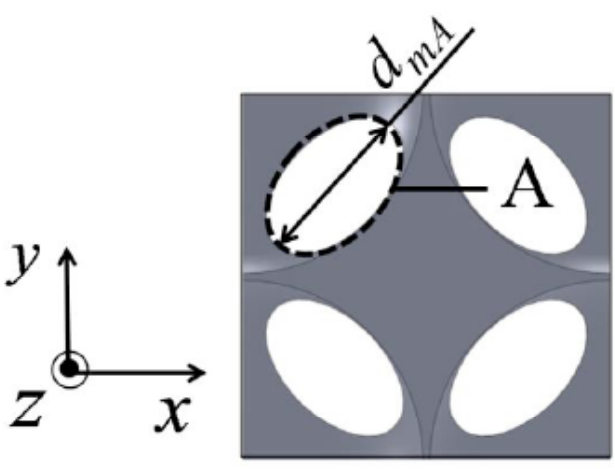

(a) $\varepsilon=0.909$

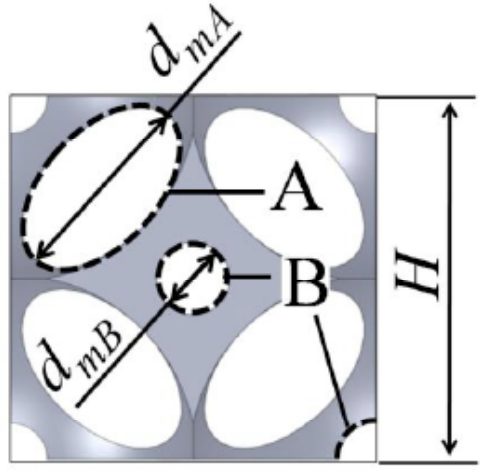

(b) $\varepsilon=0.964$

Fig. (5). Structural units observed from $z$ axis.

There are two kinds of pores A and pore B in a structural unit (see Fig. 5). Thus, the effective number of pores, namely $n$, in unit projected area $(H \times H)$ may be evaluated from

$n=\frac{n_{A} d_{m A}^{2}+n_{B} d_{m B}^{2}}{d_{m A}^{2}+d_{m B}^{2}}$

As indicated in the foregoing equation, the numbers of the pore $\mathrm{A}$ and that of the pore $\mathrm{B}$, namely $n_{A}$ and $n_{B}$, are weighted by the corresponding projected diameters $d_{m A}$ and $d_{m B}$, respectively. Note that $d_{m A}$ is much larger than $d_{m B}$ such that the effective number of pores $n$ is closer to $n_{A}(=4)$ than to $n_{B}(=2)$.

\section{Determination of Coefficients}

The macroscopic pressure gradient is modeled according to the Forchheimer extended Darcy model:

$$
-\frac{d\langle p\rangle^{f}}{d X}=\frac{\mu}{K}|\langle\vec{u}\rangle|+\rho_{f} b|\langle\vec{u}\rangle|^{2}
$$

The intrinsically averaged pressure gradient obtained from the numerical calculations is correlated against the foregoing equation to determine the permeability $K$ and Forchheimer coefficient $b$. The details of this procedure may be found elsewhere (5).

The macroscopic heat transport equation for the fluid phase is given as follow:

$$
\begin{array}{r}
\rho_{f} c_{p f} \varepsilon\langle\vec{u}\rangle \cdot \nabla\langle T\rangle^{f}=\nabla \cdot\left(\varepsilon k_{f} \nabla\langle T\rangle^{f}+\frac{1}{V} \int_{A_{\text {ivt }}} k_{f} T d \vec{A}\right) \\
-\varepsilon \rho_{f} c_{p f} \nabla \cdot\left\langle T^{\prime} \vec{u}^{\prime}\right\rangle^{f}+\frac{1}{V} \int_{A_{\text {int }}} k_{f} \nabla T \cdot d \vec{A}
\end{array}
$$

The second term on the right hand side of the equation is modeled using the thermal dispersion tensor $\overline{\overline{k_{d i s}}}$ :

$\varepsilon \rho_{f} c_{p f}\left\langle T^{\prime} \vec{u}^{\prime}\right\rangle^{f}=-\varepsilon \overline{\overline{k_{d i s}}} \cdot \nabla\langle T\rangle^{f}$

In this study, only the axial component $k_{d i s X X}$ is focused as follows:

$\frac{k_{\text {dis } X X}}{k_{f}}=\frac{\frac{r_{f} c_{p f}}{V_{f} k_{f}} \int_{V_{f}}\left(T-\langle T\rangle^{f}\right)\left(\vec{u}-\langle\vec{u}\rangle^{f}\right) d V}{d\langle T\rangle^{f} / d X}$.

$(\cos \alpha \cos \beta \overrightarrow{\mathrm{i}}+\sin \alpha \overrightarrow{\mathrm{j}}+\cos \alpha \sin \beta \overrightarrow{\mathrm{k}})$

The third term on the right hand side of equation (13) is modeled using the volumetric heat transfer coefficient $h_{v}$ as follows:

$\frac{1}{V} \int_{A_{i n t}} k_{f} \tilde{N} T d A=h_{v}\left(\langle T\rangle^{s}-\langle T\rangle^{f}\right)$

\section{RESULTS AND DISCUSSION}

The dimensionless permeability thus determined is plotted against the porosity in Fig. (6a). In the same figure, the empirical correlation [9] is plotted along with specific surface variation. The permeability increases with the porosity in accord with the empirical correlation, since the specific surface area decreases resulting in the reduction of interstitial viscous force.

In Fig. (6b), the effect of porosity on the Forchheimer coefficient is presented with the empirical correlation [10]. The ratio of the diameter of pore A to the length of numeri- 


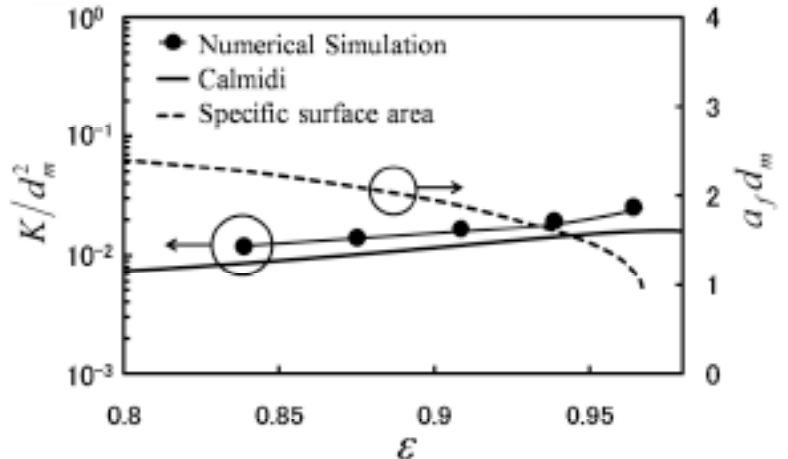

(a) Permeability

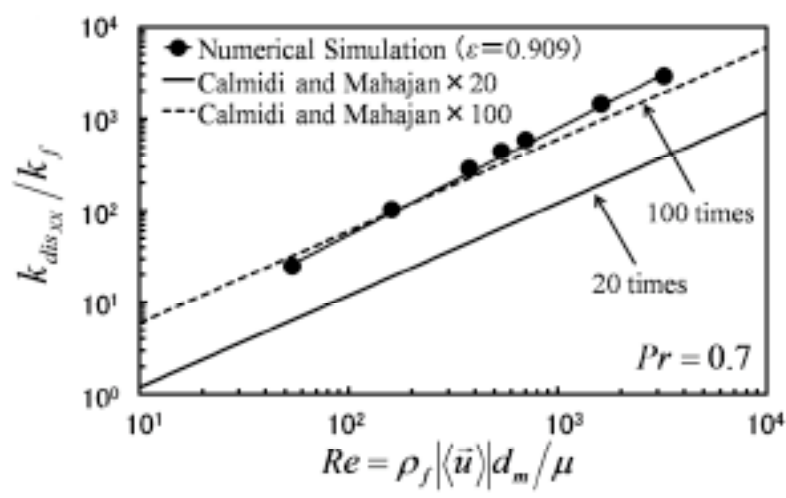

(c) Axial thermal dispersion conductivity

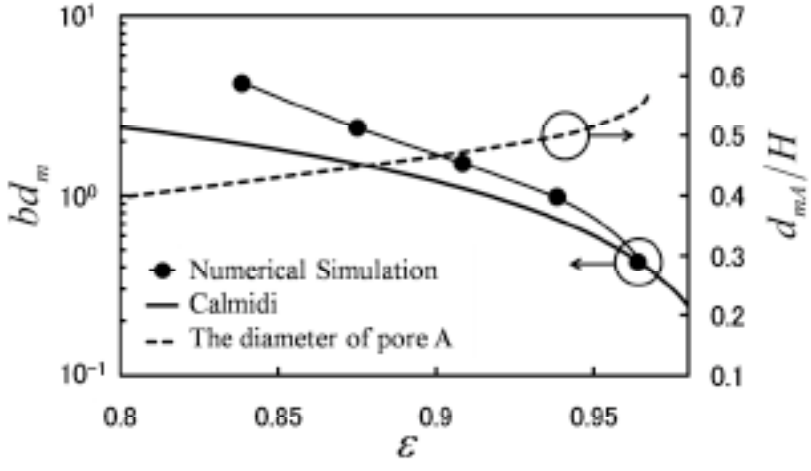

(b) Forchheimer coefficient

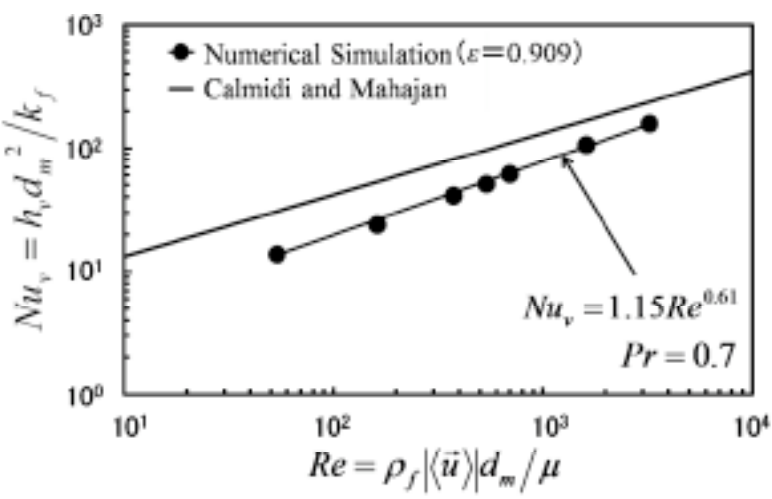

(d) Volumetric heat transfer coefficient

Fig. (6). Numerical results.

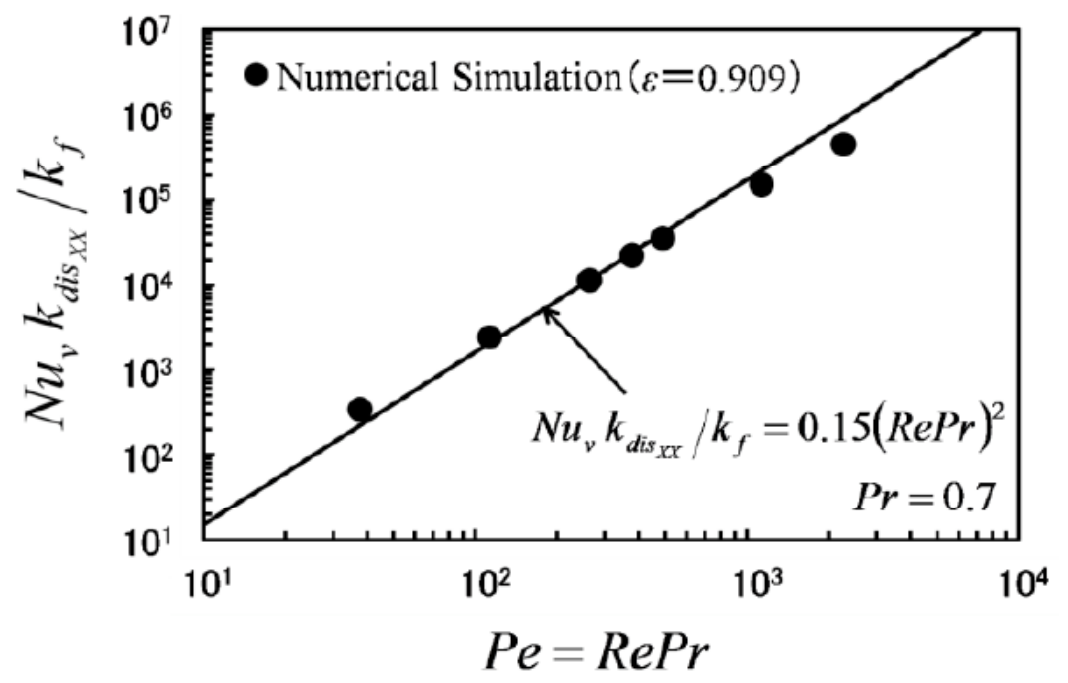

Fig. (7). Relationship between the axial thermal dispersion conductivity and the volumetric heat transfer coefficient.

cal domain $d_{m A} / H$ is also indicated in the same figure. The Forchheimer coefficient decreases as increasing the porosity, since $d_{m A} / H$ increases the porosity diminishing the interstitial form drag.

The axial thermal dispersion conductivity $k_{d i s X X}$ is plotted in Fig. (6c), for $\varepsilon=0.909$. The axial component $k_{d i s X X}$ is typically 20 to 100 times larger than the vertical one $k_{d i s Y Y}$. Thus, the empirical correlation [11] for $k_{d i s Y Y}$ is multiplied by 20 and 100 times, and plotted in the figure for comparison. The present numerical results agree well with the line generated by $k_{d i s Y Y} \times 100$. The exponent for the Reynolds number of present numerical results is found to be 1.17 , somewhat larger than 1.

Fig. (6d) shows the dimensionless volumetric heat transfer coefficient, namely Nusselt number $N u_{v}$, with the empirical correlation [11], for $\varepsilon=0.909$. From the figure, interstitial heat transfer is seen promoted as increasing Reynolds number. The volumetric heat transfer coefficient appears to be proportional to Reynolds number to the power 0.61 . 
All heat and fluid flow characteristics such as permeability, Forchheimer coefficient, axial thermal dispersion conductivity and volumetric heat transfer coefficient well agree with empirical correlations. These results clearly indicate that the present numerical method and the effective pore diameter concept are valid.

The following relation holds between the interstitial heat transfer coefficient and thermal dispersion [12]:

$$
N u_{v} k_{d i s_{X X}} / k_{f}=C(\operatorname{RePr})^{2}
$$

where $C$ is the proportional constant. Fig. (7) shows the product $N u_{v} k_{d i s X X} / k_{f}$ against the Pécret number, for $\varepsilon=$ 0.909 . The proportional constant $C=0.15$ has been determined from the numerical results. The figure clearly indicates that the proportional relationship [12] holds over a wide range of the Peclet number.

\section{CONCLUSIONS}

In this study, we carried out numerical investigation of heat and fluid flow though metal foams. The numerical results were processed to determine permeability, Forchheimer coefficient, thermal dispersion conductivity and volumetric heat transfer coefficient. The effective pore diameter concept has been proposed to correlate the resulting heat and fluid flow characteristics with available empirical correlations. Present numerical results were well accord with available empirical correlations. Moreover, the interesting relationship between the axial thermal dispersion conductivity and the volumetric heat transfer coefficient held over wide range of Pécret number. These results indicate that the present numerical method and the effective pore diameter concept are valid.

\section{NOMENCLATURE}

$A_{\text {int }}=$ interfacial area between fluid and solid phases

$b=$ Forchheimer coefficient

$c_{p} \quad=$ constant pressure specific heat

$D \quad=$ diameter of sphere

$d_{m} \quad=\quad$ the effective pore diameter

$d_{m A}, d_{m B}=$ projected diameters of pore $\mathrm{A}$ and that of pore B

$h_{v} \quad=\quad$ volumetric heat transfer coefficient

$H=$ length of numerical domain

$\vec{i}, \vec{j}, \vec{k} \quad=\quad$ unit vector in the $x, y, z$ directions

$k=$ thermal conductivity

$\overline{\overline{k_{d i s}}}=\quad$ thermal dispersion conductivity tensor

$k_{\text {dis } X X}=$ axial thermal dispersion conductivity

$K=$ permeability

\section{GREEK SYMBOLS}

$\alpha, \beta=$ angles between the macroscopic velocity vector and principal axis

\begin{tabular}{|c|c|c|}
\hline$\varepsilon$ & $=$ & porosity \\
\hline$\mu$ & $=$ & viscosity \\
\hline$\rho$ & $=$ & density \\
\hline$\tau$ & $=$ & similarity factor \\
\hline$n$ & $=$ & the effective number of pores \\
\hline$n_{A}, n_{B}$ & $=$ & $\begin{array}{l}\text { the number of the pore } A \text { and that of the } \\
\text { pore } B\end{array}$ \\
\hline$N u_{v}$ & $=$ & $\begin{array}{l}\text { dimensionless volumetric heat transfer } \\
\text { coefficient: Nusselt number }\end{array}$ \\
\hline $\mathrm{Pe}$ & $=$ & Peclet number \\
\hline $\operatorname{Pr}$ & $=$ & Prandtl number \\
\hline$R e$ & $=$ & Reynolds number \\
\hline$T$ & $=$ & temperature \\
\hline$u, v, w$ & $=$ & $\begin{array}{l}\text { velocity components in the } x, y, z \text { direc- } \\
\text { tions }\end{array}$ \\
\hline$\vec{u}$ & $=$ & velocity vector \\
\hline$V$ & $=$ & volume \\
\hline$x, y, z$ & $=$ & cartesian coordinates \\
\hline$X$ & $=$ & axis in the macroscopic flow direction \\
\hline
\end{tabular}

\section{SUBSCRIPTS}

$$
\begin{aligned}
f & =\text { fluid phase } \\
s & =\text { solid phase } \\
, \quad & =\text { deviation from volume averaged value }
\end{aligned}
$$

\section{SPECIAL SYMBOLS}

$$
\begin{array}{ll}
\langle\rangle & =\text { volume average } \\
\langle\rangle^{\prime} & =\text { intrinsic average }
\end{array}
$$

\section{CONFLICT OF INTEREST}

The authors confirm that this article content has no conflicts of interest.

\section{ACKNOWLEDGEMENT}

Declared none.

\section{REFERENCES}

[1] Z.W. Caliot, C. G. Flamant, and Z. Wang, "Numerical simulation of convective heat transfer between air flow and ceramic foams to optimize volumetric solar receiver performances", International Journal of Heat and Mass Transfer, vol. 54, pp. 1527-1537, 2011.

[2] A. Kopanidis, A. Theodorakakos, E. Gavaises, and D. Bouris, "3D numerical simulation of flow and conjugate heat transfer through a pore scale model of high porosity open cell metal foam", International Journal of Heat and Mass Transfer, vol. 53, pp. 2539-2550, 2010.

[3] S. Krishnan, S. V. Garimella, and J. Y. Murthy, "Simulation of Thermal Transport in Open-Cell Metal Foams: Effect of Periodic Unit-Cell Structure", ASME Journal of Heat Transfer, vol. 130, pp. 024503-1 024503-5, 2008.

[4] W. Xu, H. Zhang, Z. Yang, and J. Zhang, "Numerical investigation on the flow characteristics and permeability of three-dimensional 
reticulated foam materials", Chemical Engineering Journal, vol. 140, no. 1, pp. 562-569, 2008.

[5] A. Nakayama, F. Kuwahara, Y. Kawamura, and H. Koyama, "Three-Dimensional Numerical Simulation of Flow Through a Microscopic Porous Structure", In: Proceedings ASME/JSME Thermal Engineering Conference, Hawaii, 1995, vol. 3, pp. 313-318, 1995.

[6] L. Kelvin, "On the Division Of Space With Minimum Partitional Area", Philosophical Magazine, vol. 24, No. 151, pp. 503-514, 1887

[7] J. T. Richardson, Y. Peng, and D. Remue, "Properties of ceramic foam catalyst supports: pressure drop", Applied Catalysis A: General, vol. 204, pp. 19-32, 2000.

[8] X. Fu, R. Viskanta, and J. P. Gore, "Measurement and correlation of volumetric heat transfer coefficients of cellular ceramics", Experimental Thermal and Fluid Science, vol. 17, pp. 285-293, 1998.
[9] V. V. Calmidi, "Transport phenomena in high porosity fibrous metal forms", Ph.D. dissertation, University of Colorado, Boulder, CO, 1998.

[10] N. Dukhan, "Correlations for the pressure drop for flow through metal foam", Experiments in Fluids, vol. 41, no. 4, pp. 665-672, 2006.

[11] V. V. Calmidi, and R. L. Mahajan, "Forced convection in high porosity metal foams", ASME Journal of Heat Transfer, vol. 122, pp. 557-565, 2000.

[12] Y. Sano, F. Kuwahara, M. Mobedi, and A. Nakayama, "Effects of thermal dispersion on heat transfer in cross-flow tubular heat exchangers", International Journal of Heat and Mass Transfer, vol. 48, no 1, pp. 183-189, 2012

Received: March 13, 2013

(C) Kuwahara and Fumoto; Licensee Bentham Open.

This is an open access article licensed under the terms of the Creative Commons Attribution Non-Commercial License (http://creativecommons.org/licenses/by-nc/3.0/) which permits unrestricted, non-commercial use, distribution and reproduction in any medium, provided the work is properly cited. 